\title{
Variation in Anisotropy and Diffusivity along the Medulla Oblongata and the Whole Spinal Cord in Adolescent Idiopathic Scoliosis: A Pilot Study Using Diffusion Tensor Imaging
}

\author{
Y. Kong, L. Shi, S.C.N. Hui, D. Wang, M. Deng, W.C.W. Chu, and J.C.Y. Cheng
}

\begin{abstract}
BACKGROUND AND PURPOSE: Disturbed somatosensory evoked potentials have been demonstrated in patients with adolescent idiopathic scoliosis (but this functional delay was found to originate above the C5-6 level, while the lower cord level was unaffected). Together with MR imaging observation of tonsillar ectopia and a relatively tethered cord, we hypothesized that there is disturbed mean diffusivity integrity along the spinal cord. In this study, advanced DTI was used to evaluate whether there was underlying decreased WM integrity within the brain stem and spinal cord in adolescent idiopathic scoliosis and any relationship to cerebellar tonsillar ectopia. Clinical impact on balance testing was also correlated.
\end{abstract}

MATERIALS AND METHODS: Thirteen girls with adolescent idiopathic scoliosis with right thoracic curves were compared with 13 age-matched healthy girls. DTI of the brain and whole spinal cord was performed. ROIs were manually defined for the medulla oblongata and along each intervertebral segment of the cord. Mean values of fractional anisotropy and mean diffusivity were computed at the defined regions. Between-group comparisons were performed by 1-way ANOVA.

RESULTS: Significantly decreased fractional anisotropy values and increased mean diffusivity values were found at the medulla oblongata and $\mathrm{Cl}-2, \mathrm{C2}-3, \mathrm{C} 3-4$, and C4-5 segments in patients with adolescent idiopathic scoliosis compared with healthy subjects. No significant difference was found in the lower cord levels. Significant correlation was found between the tonsillar level and fractional anisotropy value at the C4-5 level in patients with adolescent idiopathic scoliosis only.

CONCLUSIONS: The findings from this study are in agreement with previous findings showing abnormal somatosensory evoked potential readings occurring only above the C5-6 level in patients with adolescent idiopathic scoliosis; these findings might partially explain the pathophysiology of the neural pathway involved.

ABBREVIATIONS: AIS = adolescent idiopathic scoliosis; FA = fractional anisotropy; MD = mean diffusivity; SEP = somatosensory evoked potential; SOT = sensory organization test

A dolescent idiopathic scoliosis (AIS) is a complex 3D deformity of the spine that affects approximately $4 \%$ of school children worldwide. ${ }^{1}$ Despite intensive research in the past de-

Received December 4, 2013; accepted after revision January 22, 2014.

From the Departments of Imaging and Interventional Radiology (Y.K., L.S., S.C.N.H., D.W., M.D., W.C.W.C.), Biomedical Engineering and Shun Hing Institute of Advanced Engineering (D.W.), and Orthopaedics and Traumatology (I.C.Y.C.), The Chinese University of Hong Kong, Shatin, New Territories, Hong Kong, China; Shenzhen Institutes of Advanced Technology (L.S.), Chinese Academy of Sciences, Shenzhen, China; and The Chinese University of Hong Kong Shenzhen Research Institute (D.W.), Shenzhen, China.

This work was supported by grants from the Research Grants Council of the Hong Kong Special Administrative Region, China (Project No. The Chinese University of Hong Kong 411910, 411811, 475711, SEG_CUHK02); grants from the National Natural Science Foundation of China (Project No. 81271653 and 81101111), a project BMEp2-13 of the Shun Hing Institute of Advanced Engineering, The Chinese University of Hong Kong; and a grant from Shenzhen Science and Technology Innovation Committee (Project No. JCYJ20120619152326449).

Please address correspondence to Defeng Wang (for technical issues), Department of Imaging and Interventional Radiology, The Chinese University of Hong Kong, Shatin, cades, the pathophysiology of AIS remains uncertain. ${ }^{2}$ There is, however, growing evidence suggesting that an underlying neurologic disorder may be a causative factor of AIS. ${ }^{3}$ Previous studies have reported changes in central nervous system structures in patients with AIS based on advanced medical imaging modalities. ${ }^{4-6}$ Furthermore in a number of MR imaging studies, the observable changes in morphologic shape and position of the cord, ${ }^{7}$ mismatch in lengthening between the cord and vertebral column, ${ }^{8}$ and increased incidence of low-lying cerebellar tonsils ${ }^{9}$ have led to the postulation of the presence of subclinical tethering of the spinal cord in AIS. ${ }^{10}$

\footnotetext{
New Territories, Hong Kong; e-mail address: dfwang@cuhk.edu.hk; and Winnie C.W. Chu (for clinical issues), Department of Imaging and Interventional Radiology, The Chinese University of Hong Kong, Shatin, New Territories, Hong Kong; Email: winnie@med.cuhk.edu.hk

- Indicates open access to non-subscribers at www.ajnr.org

http://dx.doi.org/10.3174/ajnr.A3912
}

AJNR Am J Neuroradiol 35:1621-27 Aug 2014 www.ajnr.org 

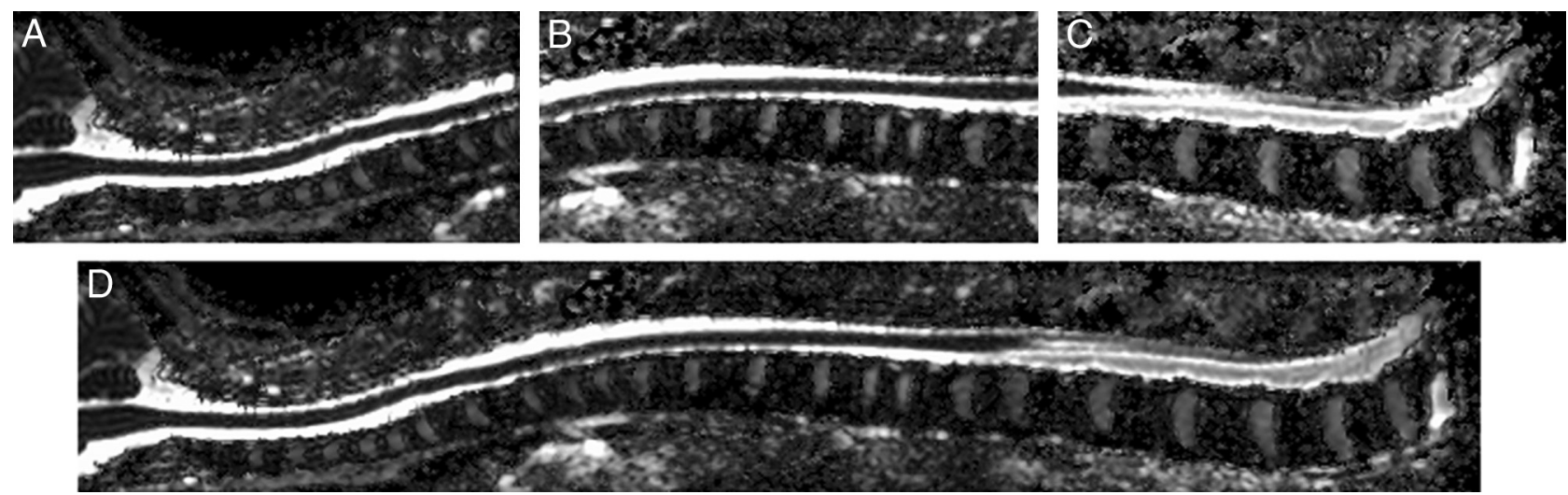

FIG 1. Sagittal mean diffusivity images show an example of spinal cord DTI stitching. Upper part (A), middle part (B), lower part (C), and stitched (D) spinal cord DTI of the whole spinal cord.

The proposed disturbed spinal cord function in AIS was further supported by the clinical observation of abnormal somatosensory function, which has been widely reported in this group. Prolonged latency or absent waveforms in posterior tibial nerve somatosensory evoked potentials (SEPs) were reported in $12 \%-$ $61 \%$ of patients with scoliosis in different series. ${ }^{8,11-13}$ A previous study also suggested that balance control was affected by somatosensory input. ${ }^{14}$ In the study by Cheng et al, ${ }^{15} 14.6 \%$ of patients with AIS had abnormal SEPs with either prolonged latency or decreased amplitude, while tonsillar ectopia was found in 33.3\% of those with abnormal SEPs. A subsequent study with detailed analysis of SEPs recorded at 3 different levels (popliteal fossa, cervical level C4-6, and the scalp) showed that in patients with AIS with abnormal SEPs, the readings were only abnormal at the scalp level, while the readings at the popliteal and cervical regions were normal, indicating that the level of abnormality along the somatosensory pathway was superior to that at the cervical C5-6 level. ${ }^{16}$

DTI is a recent advanced imaging technique to evaluate WM architecture within the brain and spinal cord in vivo. ${ }^{17}$ This technique can detect the microstructural changes of the WM and is presently a promising tool to study WM fiber bundles in vivo. In recent years, the advanced technique has been increasingly used to examine the spinal cord parenchyma for multiple diseases, including myelitis, spinal cord injury, ${ }^{18}$ multiple sclerosis, ${ }^{19}$ and intramedullary spinal cord neoplasms. ${ }^{20}$ On the basis of the observation of tonsillar ectopia, relatively tethered cord, and abnormal SEP, we hypothesized that there is disturbed WM integrity within the brain stem and the spinal cord, most likely at the more cranial level as indicated by the level of SEP abnormality in the previous study. ${ }^{16}$ The disturbed WM integrity is likely reflecting underlying cord abnormality in patients with AIS in addition to their skeletal abnormality. We sought to prove the above hypothesis by evaluating the fractional anisotropy (FA) and mean diffusivity (MD) values of the spinal cord and correlating them with the position of cerebellar tonsils and balance testing.

\section{MATERIALS AND METHODS \\ Subjects}

Thirteen girls with AIS and 13 age-matched healthy girls were recruited. The AIS group consisted of patients with a dominant right thoracic curve (the most common curve type in AIS) from 11 to 16 years of age (mean, 13.6 years of age). The Cobbs angle ranged from $16^{\circ}$ to $37^{\circ}$ (mean, $24.67^{\circ}$ ). They were consecutively recruited from our outpatient clinic. The healthy controls were recruited from local schools and were from 12 to 15 years of age (mean, 13.9 years of age). Inclusion criteria of AIS were the following: 1) age between 12 and 18 years, 2) right thoracic curves, 3) availability of medical history from the parents or legal guardians. Inclusion criteria of healthy controls were the following: 1) age between 12 and 18 years, and 2) no signs of scoliosis from the annual assessment by the Student Health Service. Exclusion criteria for both patients with AIS and healthy controls included any history of head or back injury; neurologic symptoms such as headache, weakness, or numbness in any limbs; urinary incontinence; nocturnal enuresis; or any space-occupying lesion found on screening MR imaging. All participants were right-handed Chinese adolescents with normal neurologic examination findings. Approval was obtained from the ethics committee of the hospital and university.

\section{MR Imaging Data Acquisition}

All MR imaging examinations were performed on the brain and spinal cord for each subject by using a 3T scanner (Achieva TX series; Philips Healthcare, Best, the Netherlands). An 8-channel sensitivity encoding head coil was used to acquire T1-weighted images and DTI covering the whole brain. Brain T1-weighted images were obtained by using a 3D fast-field echo imaging sequence with the following parameters: $\mathrm{TR}=18 \mathrm{~ms}, \mathrm{TE}=2.4 \mathrm{~ms}$, FOV $=210 \times 210 \mathrm{~mm}^{2}$, flip angle $=30^{\circ}, \mathrm{NEX}=1$, matrix $=$ $232 \times 232$, section $=200$. Brain DTI was performed by using the single-shot EPI sequence with the following parameters: $\mathrm{TR}=$ $8667 \mathrm{~ms}, \mathrm{TE}=60 \mathrm{~ms}, \mathrm{FOV}=224 \times 224 \mathrm{~mm}^{2}$, flip angle $=90^{\circ}$, $\mathrm{NEX}=1$, matrix $=112 \times 109$, section $=70$, section thickness $=$ $2 \mathrm{~mm}$, gap $=2 \mathrm{~mm}$. After reconstruction, images were zeropadded and interpolated to $224 \times 224$ with a voxel size of $1 \times 1 \times$ $2 \mathrm{~mm}^{3}$. Thirty-two directions of diffusion gradients with b-value of $1000 \mathrm{~s} / \mathrm{mm}^{2}$ and $1 \mathrm{~B} 0$ volume were collected.

A 15-channel sensitivity encoding spine coil was used to scan the spinal cord for each subject. Conventional T2weighted images were acquired with a turbo spin-echo sequence with the following parameters: $\mathrm{TR}=4048 \mathrm{~ms}$, $\mathrm{TE}=$ 


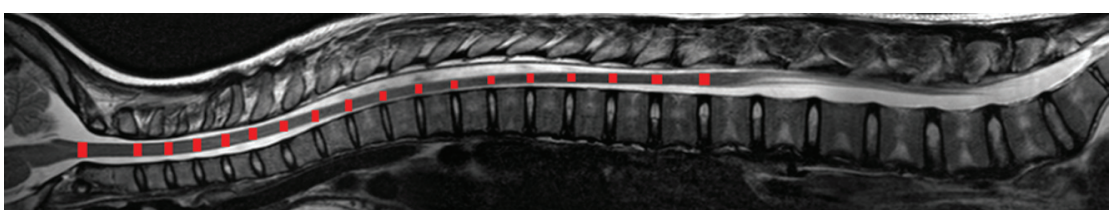

FIG 2. Regions of interest of the spinal cord at different intervertebral disk levels.

\section{Quantification of Fiber Integrity of the Spinal Cord}

For each subject, diffusion tensor images of the spinal cord were adjacently stitched together to produce the DTI of the entire spinal cord. ${ }^{22}$ In brief, the adjacent diffusion tensor images were first aligned by using a feature-based regis-

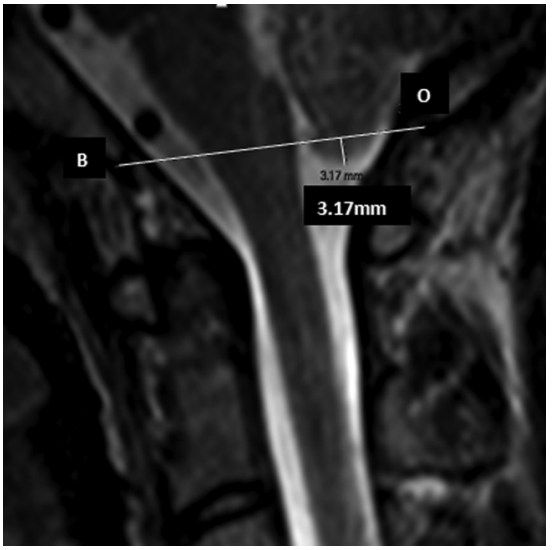

FIG 3. The basion and opisthion line and the measurement of the cerebellar tonsil level.

$120 \mathrm{~ms}$, flip angle $=90^{\circ}, \mathrm{NEX}=2$. Spinal cord DTI was acquired at the upper, middle, and lower parts with sufficient overlapping areas. Figures $1 A-C$ show an example of these 3 parts. In each part, sagittal sections parallel to the long axis of the subject's body were acquired to cover the whole spinal cord. Thirty sagittal sections were collected for the healthy girls in each part. Due to the curves of spinal cord in AIS, an equal or larger number of sagittal sections, ranging from 30 to 40, were required to capture the entire spinal cord. Spinal cord DTI was performed by using the single-shot EPI sequence with the following parameters: $\mathrm{TR}=8180 \mathrm{~ms}, \mathrm{TE}=63 \mathrm{~ms}, \mathrm{FOV}=130 \times$ $219 \mathrm{~mm}^{2}$, flip angle $=90^{\circ}, \mathrm{NEX}=6$, matrix $=64 \times 110$, section thickness $=2 \mathrm{~mm}$, gap $=2 \mathrm{~mm}$. After reconstruction, images were zero-padded and interpolated to $224 \times 224$ with voxel size of $0.98 \times 0.98 \times 2 \mathrm{~mm}^{3}$. Six directions of diffusion gradients with a b-value of $500 \mathrm{~s} / \mathrm{mm}^{2}$ and $1 \mathrm{~B} 0$ volume were collected. The number of gradients was reduced compared with the brain DTI because of time constrains because DTI acquisition of the whole spinal cord required 3 separate sessions. Together with the morphologic T2-weighted sequences, the total scanning time of the spinal cord was 30 minutes or longer if the scoliosis curve was severe. The scanning time was, therefore, kept to a minimum to avoid motion artifacts and discomfort of the subject while image quality was acceptable for analysis.

\section{MR Image Preprocessing}

The FMRIB Diffusion Toolbox (http://www.fmrib.ox.ac.uk/fsl/ $\mathrm{fdt} /$ index.html ${ }^{21}$ was used to perform preprocessing of brain and spinal cord DTI datasets. Distortion correction was first performed to remove eddy currents and motion artifacts. Diffusion tensors and parameters, including FA, colored FA, and MD, were then computed in the Diffusion Toolbox by using the robust weighted least-squares fitting method. tration algorithm. All the images were then warped to the same space and were stitched together by using an effective feathering approach with the Log-Euclidean metrics. The FA and MD scalar maps were visualized to evaluate the effectiveness of the stitching results. Figure 1 shows an MD map of the stitched spinal cord DTI from 1 healthy subject.

\section{Region-of-Interest Definition of FA and MD for the Medulla Oblongata and Spinal Cord}

A commonly used method was adopted to evaluate the mean value of the DTI metrics on the whole medulla oblongata. ${ }^{23}$ The medulla oblongata was first manually defined on the high-resolution T1-weighted image. The inferior margin was defined at the level of the foramen magnum, while the superior margin was defined at the level of the bulbopontine sulcus. The defined region was mapped to DTI space by affine-registering the T1 image to the B0 image by using a linear image registration approach ${ }^{24}$

A segmental approach was used to obtain different ROIs throughout the spinal cord because it was not feasible to adopt a voxel-by-voxel approach in view of the anatomy of the cord and the intrinsic limitation that the high-dimensional tensor structure of DTI could not be directly used for analysis. The spinal cord was segmented and numbered according to its corresponding vertebral levels from $\mathrm{C} 2$ to $\mathrm{L} 1$. The FA and MD values were then measured at different intervertebral disk levels (ranging from C1/2 to T12/L1, shown in Fig 2). Eighteen ROIs were obtained in each subject. ROIs were manually drawn on FA maps at every axial section at designated levels along the whole spinal cord. To ensure the proper anatomic localization, an experienced research associate with neuroradiology training was responsible for identifying and drawing the ROIs. Besides, special attention was paid to avoid CSF and the gradient inhomogeneity effects in the region-of-interest selections. ${ }^{19}$ Each region of interest included approximately 50 voxels at each level. With the resolution of $0.98 \times 0.98 \times 2$ $\mathrm{mm}^{3}$, the volumes were approximately $100 \mathrm{~mm}^{3}$. Special attention was made to avoid partial volume effects from the CSF during the selection of ROIs.

\section{Measurement of Cerebellar Tonsil Level}

Because low-lying tonsils have been commonly reported in AIS and this finding might be related to the severity of cord tethering and might exert some compressive effect on the brain stem/cervical cord, the position of the cerebellar tonsil was assessed on the midsagittal image according to the method described in previous studies. ${ }^{8,15,25}$ The level of the cerebellar tonsil relative to a reference line connecting the basion and opisthion line was measured according to the method described by Aboulezz et al (Fig 3). ${ }^{26}$ The distance measured from above the basion and opisthion line was conventionally assigned as a positive value, while the distance

AJNR Am J Neuroradiol 35:1621-27 Aug 2014 www.ajnr.org 1623 

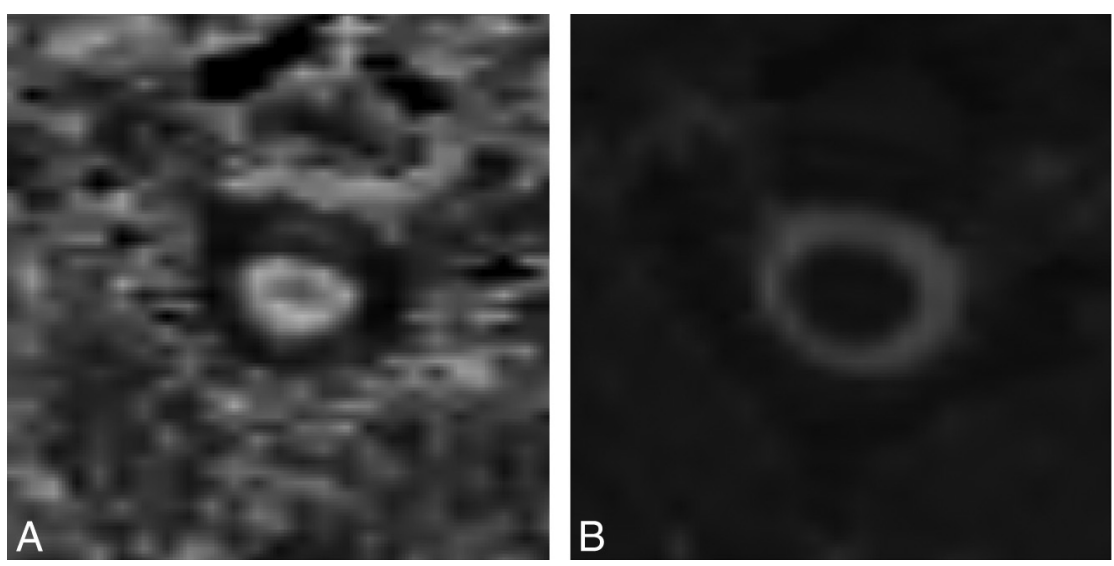

FIG 4. Fractional anisotropy and mean diffusivity maps of 1 axial section at C2-3 from 1 healthy control.

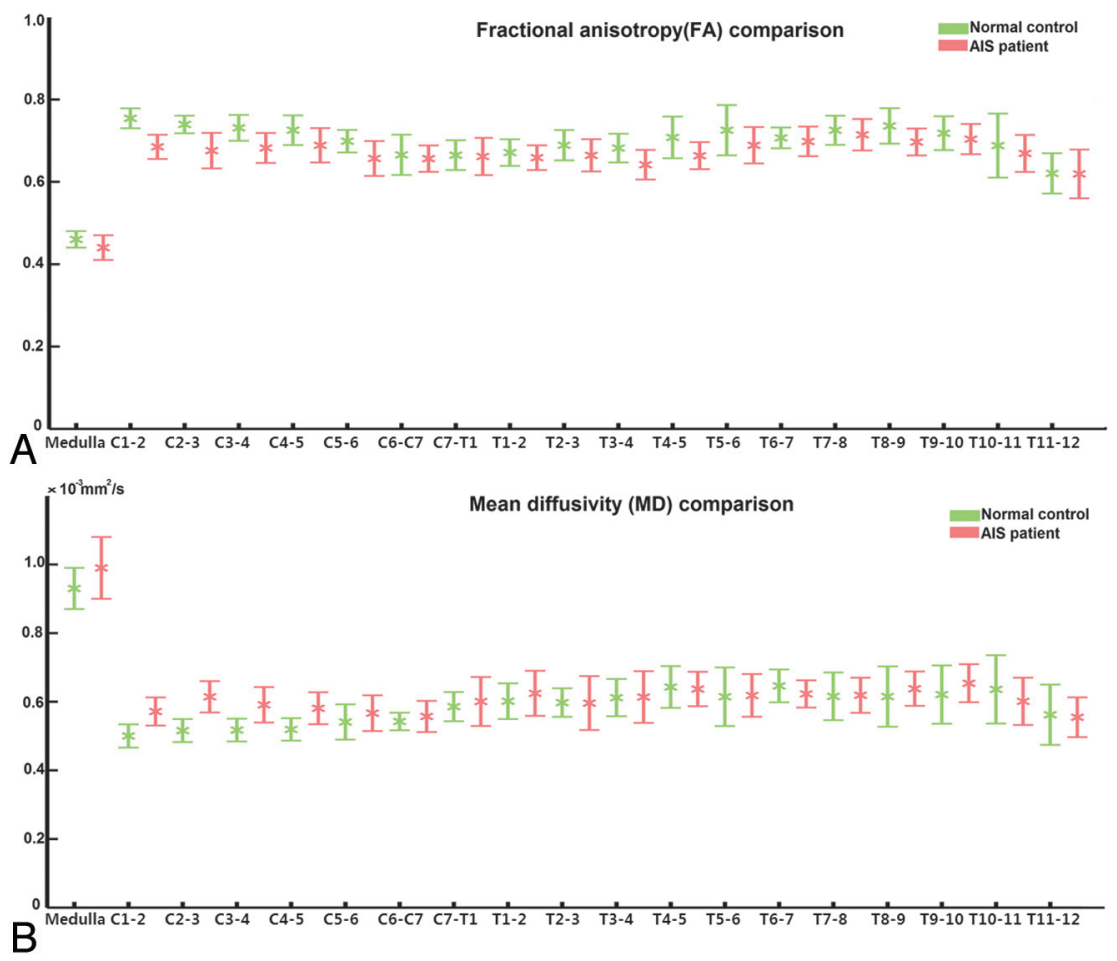

FIG 5. Fractional anisotropy $(A)$ and mean diffusivity $(B)$ values for regions of interest at different levels of the medulla oblongata and spinal cord for healthy controls and patients with adolescent idiopathic scoliosis. (ranging from 0.86 to 0.99 ). The $\mathrm{MD}$ mean value was 0.85 (ranging from 0.79 to 0.96$)$.

The level of the cerebellar tonsils was independently measured by 2 observers (1 radiologist and 1 research associate who had 3 years of experience in MR imaging measurement). The intraclass correlation coefficient was 0.89 .

\section{Postural Balance Study}

Smart Equitest-Computerized Dynamic Posturography (NeuroCom International, Clackamas, Oregon) was used for the study. A standardized sensory organization test (SOT) ${ }^{27}$ protocol was used to identify objectively any abnormalities in the postural control. The SOT measured how well a patient could maintain their postural stability under 6 sensory conditions: 1) normal vision, firm surface; 2) eyes closed, firm surface; 3 ) sway-referenced visual surroundings, firm surface; 4) normal vision, swayreferenced surface; 5) eyes closed, sway-referenced surface; and 6) swayreferenced visual surroundings, swayreferenced surface. The equilibrium score quantified the center of gravity sway or postural stability under each of the 3 trials of the 6 sensory conditions. ${ }^{14}$

\section{Statistical Analysis}

Between-group comparisons of FA and $\mathrm{MD}$ values were performed at the medulla oblongata and all the segments by using the 1-way ANOVA test and the false discovery rate, with $P=.05$ used to correct the multiple comparisons. Due to a small sample size, a Spearman correlation was performed to look for relationships among FA/MD values, tonsillar level, and the SOT score. measured below the basion and opisthion line was assigned as a negative value.

\section{Measurement Reproducibility}

For FA and MD assessment of the medulla oblongata and spinal cord at different levels, we strictly obeyed the precise anatomic definition for the ROIs. Figure 4 shows the FA and MD maps of 1 axial section at the C2-3 level from 1 healthy control. The boundary can be manually recognized without difficulty. The intraclass correlation coefficients of intraobserver variation for the medulla oblongata and different segments of the spinal cord were measured for FA and MD to test the reproducibility of the measurements. The mean intraclass correlation coefficient for FA was 0.91

\section{RESULTS \\ FA and MD Values in the Medulla Oblongata and Spinal Cord}

The mean values of FA and MD obtained from ROIs at different levels of the medulla oblongata and spinal cord were compared between the AIS and healthy control groups. The summary of FA and $\mathrm{MD}$ values (mean $\pm \mathrm{SE}$ ) at different levels is shown in Fig 5 . The FA and MD values had similar ranges between the 2 groups. The FA values ranged from 0.4 to 0.8 , while the $\mathrm{MD}$ values were in the range of $0.4-1$ $\left(\times 10^{-3} \mathrm{~mm}^{2} / \mathrm{s}\right)$. The data above were in agreement with measurements from a previous study. ${ }^{28}$ The patterns of variation of FA and MD along the spinal cord were similar in the AIS and healthy groups. 
Table 1: Descriptive statistics of the FA comparison between AIS and controls

\begin{tabular}{lccc}
\hline & AIS & Control & $\boldsymbol{P}$ Value \\
\hline Medulla & $0.44(0.03)$ & $0.46(0.02)$ & $<.01$ \\
Cl-2 & $0.68(0.03)$ & $0.75(0.02)$ & $<.01$ \\
C2-3 & $0.68(0.04)$ & $0.74(0.02)$ & $<.01$ \\
C3-4 & $0.68(0.04)$ & $0.73(0.03)$ & $<.01$ \\
C4-5 & $0.69(0.04)$ & $0.72(0.04)$ & .03 \\
\hline
\end{tabular}

Table 2: Descriptive statistics of the MD comparison between AIS and controls

\begin{tabular}{lccc}
\hline & AIS $\left(\times 10^{-3} \mathbf{m m}^{2} / \mathbf{s}\right)$ & $\begin{array}{c}\text { Control } \\
\left(\times 10^{-3} \mathbf{~ m m}^{2} / \mathbf{s}\right)\end{array}$ & $P$ Value \\
\hline Medulla & $0.99(0.05)$ & $0.93(0.05)$ & $<.01$ \\
Cl-2 & $0.57(0.05)$ & $0.50(0.03)$ & $<.01$ \\
C2-3 & $0.60(0.06)$ & $0.52(0.03)$ & $<.01$ \\
C3-4 & $0.58(0.05)$ & $0.52(0.03)$ & $<.01$ \\
C4-5 & $0.57(0.04)$ & $0.52(0.03)$ & .01 \\
\hline
\end{tabular}

Statistical analysis revealed significant difference in FA values between the AIS and control groups $(P<.05)$. The FA values in the AIS group were significantly lower at the medulla oblongata and along the cervical cord at C1-2, C2-3, C3-4, and C4-5 levels. Significantly higher MD values were observed in all the corresponding levels when comparing the AIS group with normal controls $(P<.05)$. The results are summarized in Tables 1 and 2 . There was, however, no significant difference in FA or MD values in the cord between the 2 groups from C5-6 downward, including the entire thoracic cord. In the AIS group, a significant correlation was found between the Cobbs angle and FA at the T8-9 level $(P=.002)$, which corresponded to the level of the scoliotic apex in most of the subjects in this cohort; the FA values, however, showed no significant difference between patients with AIS and controls.

\section{Cerebellar Tonsil Level and Correlations}

The tonsil level between patients with AIS and healthy controls was analyzed. The average tonsil level was $-0.28 \mathrm{~mm}$ (range, -9.07-5.11 mm) in AIS, while it was $1.32 \mathrm{~mm}$ (range, -5.47-5.43 $\mathrm{mm}$ ) in healthy controls. A positive value indicated the tip of the tonsil above the basion and opisthion line and vice versa. Although the level of the cerebellar tonsils was lower than that in controls, this did not reach statistical significance $(P=.165)$, likely, in part, due to the small sample size.

In general when taking into account all subjects (both patients with AIS and healthy controls), there was no significant correlation between the FA values in the medulla oblongata/spinal cord and the tonsillar level. However, if only the AIS group was considered, a significant correlation was noted between the tonsillar level and FA values at $\mathrm{C} 4-5(P=.007$ and correlation coefficient $=0.707)$, while a trend toward significance was at C7-T1 $(P=.059$, correlation coefficient $=-0.536)$. No such correlation was observed in the healthy control group.

\section{SOT Findings}

For the SOT, a lower score was observed in AIS only at condition 6 (ie, sway-referenced visual surroundings, sway-referenced surface). A borderline significance was found in the correlation between the SOT core and FA values at the medulla $(P=.05)$.

\section{DISCUSSION}

A number of studies have analyzed the morphologic changes within the central nervous system in AIS. ${ }^{29,30}$ MR imaging has long been used for assessment of the brain and spinal cord in AIS in view of its high resolution and excellent soft-tissue contrast. ${ }^{10}$ Geissele et $\mathrm{al}^{31}$ found asymmetry in the ventral medulla relating to the location of the corticospinal tracts in some patients with AIS. Dohn et $\mathrm{al}^{32}$ reported a significant rotation of the spinal cord in patients with AIS. Smorgick et $\mathrm{al}^{29}$ reported the spinal cord in AIS following the curve of scoliosis on T2-weighted MR imaging. In previous works, ${ }^{7,8,30}$ significantly reduced ratios of spinal cord to vertebral column were found in patients with AIS by using multiplanar reformatted MR imaging. All the above observations were based on conventional MR imaging, which only revealed macroscopic information of the brain stem and spinal cord. DTI provides information about the anisotropy and diffusivity of WM fibers within the central nervous system and, hence, is more sensitive to pathologic changes compared with conventional MR imaging. In this pilot study, we used the DTI technique to evaluate the medulla oblongata and spinal cord of the patients with AIS at the microstructural level, which might help to understand the pathophysiology accounting for the abnormal SEP, which was commonly observed in this group.

In this study, we applied the DTI technique to examine the entire spinal cord in AIS. In previous studies, only part of the cord was examined because this was the intrinsic limitation of DTI by its relatively small FOV. ${ }^{33,34}$ In this study, we have used an effective validated in-house method, ${ }^{22}$ which enabled analysis of the entire spinal cord by stitching DTI acquired at different levels. As the diffusion tensor images of the entire spinal cord were stitched and connected together, ROIs could then be correctly positioned with reference to the vertebral and intervertebral levels.

However, there were still technical difficulties that we tried to overcome as illustrated below: First, compared with brain DTI, the small structure of spinal cord was more sensitive to distortion derived from eddy currents and physiologic artifacts. The distortion can potentially occur on DTI of both healthy controls and patients with AIS. It may lead to inaccurate calculation of diffusion tensors and scalar maps. Of note, it was even more challenging for patients with AIS compared with healthy controls. The stretching of the cord in AIS made it lie closer to the thecal sac and spinal canal over the curved segments, ${ }^{8,33}$ leading to gradient inhomogeneities in these areas. Furthermore, the ROIs were defined in an oblique manner in the coronal view at the curved segments. The powerful Diffusion Toolbox was used to correct the distortion. To minimize measurement errors due to the intrinsic limitations of DTI in the spinal cord, we did not include the voxels influenced by gradient inhomogeneities in the region-ofinterest definition. A relatively large number of voxels were selected to reduce the influence of the oblique manner of region-ofinterest selection in patients with AIS. The curves of the patients with AIS in our study began at a vertebral level lower than T6; hence, when we compared FA and MD values between patients with AIS and controls, measurements were only affected by the above factor in segments below T6, while measurements at the cervical segments were not affected.

Last, in our study, different numbers of noncollinear gradients 
were used for the brain and spinal cord DTI. A relatively small number of gradients were acquired for the spinal cord DTI compared with the brain DTI. This may affect the accuracy of the tensor estimation. To maximally reduce the influence, we applied the robust weighted least-squares fitting approach to guarantee the high image quality in the spinal cord DTI.

Significant differences in FA and MD values were found along the medulla oblongata and the cervical spinal cord in AIS. These changes were correlated with the SEP dysfunction that was reported in AIS as discussed in the introduction. Recent studies demonstrated a correlation between DTI and SEP, ${ }^{35-37}$ in which a decrease of FA values was found to be associated with abnormal SEPs. ${ }^{35,36}$ Petersen et $\mathrm{al}^{35}$ proposed that both DTI metrics and neuronal excitability were affected by morphometry and permeability of the axonal membrane to water. In our study, a decrease in FA values within the medulla oblongata and from $\mathrm{C} 1-2$ to C4 -5 cervical cord levels in AIS was in agreement with previously observed high percentage of disturbed somatosensory function in this group. Most interesting, a correlative relationship was also found between the tonsillar level and abnormal FA values in AIS. Our proposed explanation for the above changes was that there was an anatomic compression of the relatively low-set cerebellar tonsil ${ }^{9}$ onto the craniocervical junction. This subclinical type of cord compression might affect the neural pathway above and below the maximal area of compression (ie, the lower part of the brain stem [the medulla oblongata] and the upper cervical cord region). Another consideration was that the cord compression caused by the low-set tonsils could be dynamic and might not be readily reflected by the static MR images of the subjects with AIS who were scanned in a supine position. However, the tonsil might descend further and the cord compression might be more significant when the patients with AIS resume an upright position, hence causing chronic insult to the brain stem and cervical cord during the daytime. The DTI findings support our proposed hypothesis of disturbed WM integrity within the brain stem and spinal cord, which, together with low-lying cerebellar tonsils, is an associated feature of a tethered cord in AIS. The changes in FA and MD are in line with abnormal SEPs observed clinically.

One of the main limitations of our study is that we did not have SEP correlations due to equipment malfunction. However, SEPs have been well-documented and reproduced in other studies. ${ }^{8,11-15}$ We are confident that a consistent observation will be found in the current cohort if SEP testing has been performed. However, in future research, the SEP abnormalities should be further quantified and correlated with the FA/MD values to enhance a better understanding of the relationships between anatomic and electrophysiologic derangement within the cord in AIS with different curve severity and resulting cord tethering.

In this study, we tried to look at other available clinical tests for correlation. The SOT test was, therefore, chosen. A lower score at SOT condition 6 (ie, sway-referenced visual surroundings, swayreferenced surface) with borderline correlation with FA values at the medulla might not be just a causal relationship because part of the function of the medulla is body balance. The disturbed WM integrity at the medulla level might, therefore, affect the balance control, which was reflected by a lower SOT score in this particular condition 6 when the body relied heavily on brain stem input to maintain body posture; however, this observation needs to be substantiated by future large-scale studies.

Another limitation is the relatively small number of subjects in this cohort. However, our preliminary findings are encouraging, and we hope to undergo a larger-scale study if we have enough funding to pursue both SEP and DTI assessment in more subjects. Because the medulla oblongata/brain stem is the cross-road for nerve fibers involved in other higher cerebral functions, the changes on DTI may also correlate with other known neurologic abnormalities in AIS, such as abnormal postural balance or impaired vestibular function, which may be partially related to somatosensory function. Further investigations correlating DTI and other neurologic functions should be addressed in future research. The information obtained may provide a new insight to the pathophysiology of the neural pathway in AIS and may potentially affect treatment of AIS in the future.

\section{CONCLUSIONS}

We applied the DTI technique to evaluate the function of WM fibers along the medulla oblongata and spinal cord in patients with AIS. This study has demonstrated that there is altered DTI metrics in the medulla oblongata and the cervical spinal cord in patients with AIS compared with age-matched healthy subjects. Significant correlation is presented between FA values at the $\mathrm{C} 4-5$ and low-set tonsillar level. The findings from this study are in agreement with those in previous studies showing that abnormal SEP readings only occurred above the $\mathrm{C} 5-6$ level in patients with AIS and might partially explain the pathophysiology of the neural pathway involved. If substantiated, this hypothesis may potentially affect treatment of AIS in the future.

Disclosures: Youyong Kong, Lin Shi, Steve C.N. Hui, Defeng Wang, Min Deng, and Winnie C.W. Chu—RELATED: Grant: Research Grants Council of the Hong Kong Special Administrative Region, China (Project No. CUHK 411910, 411811, 475711, SEG_CUHK02), ${ }^{*}$ grants from the National Natural Science Foundation of China (Project No. 81271653 and 81101111), ${ }^{*}$ a project BME-p2-13 of the Shun Hing Institute of Advanced Engineering, The Chinese University of Hong Kong, and a grant from Shenzhen Science and Technology Innovation Committee (Project No. JCYJ20120619152326449).* Jack C.Y. Cheng-UNRELATED: Board Membership: Journal of Pediatric Orthopaedics, Comments: Editorial Board. *Money paid to the institution.

\section{REFERENCES}

1. Shi L, Wang D, Chu WC, et al. Automatic MRI segmentation and morphoanatomy analysis of the vestibular system in adolescent idiopathic scoliosis, Neuroimage 2011;54(suppl 1):S180-88

2. Burwell RG, Dangerfield PH, Freeman BJC. Concepts on the pathogenesis of adolescent idiopathic scoliosis, bone growth and mass, vertebral column, spinal cord, brain, skull, extra-spinal left-right skeletal length asymmetries, disproportions and molecular pathogenesis. In: Grivas TB, ed. Conservative Scoliosis Treatment: 1st Sosort Instructional Course Lectures Book. Amsterdam: IOS Press; 2009;135:3-52

3. Domenech J, Garcia-Marti G, Marti-Bonmati L, et al. Abnormal activation of the motor cortical network in idiopathic scoliosis demonstrated by functional MRI. Eur Spine J 2011;20:1069-78

4. Shi L, Wang D, Chu WCW, et al. Volume-based morphometry of brain MR images in adolescent idiopathic scoliosis and healthy control subjects. AJNR Am J Neuroradiol 2009;30:1302-07

5. Wang DF, Shi L, Chu WCW, et al. Abnormal cerebral cortical thinning pattern in adolescent girls with idiopathic scoliosis. Neuroimage 2012;59:935-42

6. Liu TM, Chu WC, Young G, et al. MR analysis of regional brain 
volume in adolescent idiopathic scoliosis: neurological manifestation of a systemic disease. J Magn Reson Imaging 2008;27:732-36

7. Chu WC, Man GC, Lam WW, et al. Morphological and functional electrophysiological evidence of relative spinal cord tethering in adolescent idiopathic scoliosis. Spine (Phila Pa 1976) 2008; 33:673-80

8. Chu WC, Lam WW, Chan YI, et al. Relative shortening and functional tethering of spinal cord in adolescent idiopathic scoliosis? Study with multiplanar reformat magnetic resonance imaging and somatosensory evoked potential. Spine (Phila Pa 1976) 2006;31: E19-25

9. Chu WC, Man GC, Lam WW, et al. A detailed morphologic and functional magnetic resonance imaging study of the craniocervical junction in adolescent idiopathic scoliosis. Spine 2007;32:1667-74

10. Chu WC, Rasalkar DD, Cheng JC. Asynchronous neuro-osseous growth in adolescent idiopathic scoliosis-MRI-based research. $P e$ diatr Radiol 2011;41:1100-11

11. Cheng JC, Guo X, Sher AH. Posterior tibial nerve somatosensory cortical evoked potentials in adolescent idiopathic scoliosis. Spine 1998;23:332-37

12. Hausmann ON, Boni T, Pfirrmann CWA, et al. Preoperative radiological and electrophysiological evaluation in 100 adolescent idiopathic scoliosis patients. Eur Spine J 2003;12:501-06

13. Lao ML, Chow DH, Guo X, et al. Impaired dynamic balance control in adolescents with idiopathic scoliosis and abnormal somatosensory evoked potentials. J Pediatr Orthop 2008;28:846-49

14. Guo X, Chau WW, Hui-Chan CW, et al. Balance control in adolescents with idiopathic scoliosis and disturbed somatosensory function. Spine 2006;31:E437-40

15. Cheng JC, Guo X, Sher AH, et al. Correlation between curve severity, somatosensory evoked potentials, and magnetic resonance imaging in adolescent idiopathic scoliosis. Spine 1999;24:1679-84

16. Chau WW, Guo X, Fu LLK, et al. Abnormal somatosensory evoked potential (SSEP) in adolescent with idiopathic scoliosis- the site of abnormality. In: International Research Society of Spinal Deformities Symposium. Vancouver, Canada. 2004:279-81

17. Kong $\mathrm{Y}$, Wang $\mathrm{D}$, Wang $\mathrm{T}$, et al. 3D diffusion tensor magnetic resonance images denoising based on sparse representation. In: International Conference on Machine Learning and Cybernetics. Guilin, China. 2011:1602-06

18. Konomi T, Fujiyoshi K, Hikishima K, et al. Conditions for quantitative evaluation of injured spinal cord by in vivo diffusion tensor imaging and tractography: preclinical longitudinal study in common marmosets. Neuroimage 2012;63:1841-53

19. Théaudin M, Saliou G, Ducot B, et al. Short-term evolution of spinal cord damage in multiple sclerosis: a diffusion tensor MRI study. Neuroradiology 2012;54:1171-78

20. Setzer M, Murtagh RD, Murtagh FR, et al. Diffusion tensor imaging tractography in patients with intramedullary tumors: comparison with intraoperative findings and value for prediction of tumor resectability. J Neurosurg Spine 2010;13:371-80

21. Jbabdi S, Sotiropoulos SN, Savio AM, et al. Model-based analysis of multishell diffusion MR data for tractography: how to get over fitting problems. Magn Reson Med 2012;68:1846-55

22. Wang D, Kong Y, Shi L, et al. Fully automatic stitching of diffusion tensor images in spinal cord. J Neurosci Methods 2012;209:371-78

23. Zhao DD, Zhou HY, Wu QZ, et al. Diffusion tensor imaging characterization of occult brain damage in relapsing neuromyelitis optica using 3.0 T magnetic resonance imaging techniques. Neuroimage 2012;59:3173-77

24. Jenkinson M, Smith S. A global optimisation method for robust affine registration of brain images. Medical Image Anal 2001; 5:143-56

25. Cheng JCY, Chau WW, Guo X, et al. Redefining the magnetic resonance imaging reference level for the cerebellar tonsil: a study of 170 adolescents with normal versus idiopathic scoliosis. Spine 2003;28:815-18

26. Aboulezz AO, Sartor K, Geyer CA, et al. Position of cerebellar tonsils in the normal population and in patients with Chiari malformation: a quantitative approach with MR imaging. J Comput Assist Tomogr 1985;9:1033-36

27. Brouwer B, Culham EG, Liston RA, et al. Normal variability of postural measures: implications for the reliability of relative balance performance outcomes. Scand J Rehabil Med 1998;30:131-37

28. Cui J, Wen CY, Hu Y, et al. Orientation entropy analysis of diffusion tensor in healthy and myelopathic spinal cord. Neuroimage 2011;58:1028-33

29. Smorgick Y, Settecerri JJ, Baker KC, et al. Spinal cord position in adolescent idiopathic scoliosis. J Pediatr Orthop 2012;32:500-03

30. Chu WC, Lam WM, Ng BK, et al. Relative shortening and functional tethering of spinal cord in adolescent scoliosis: result of asynchronous neuro-osseous growth, summary of an electronic focus group debate of the IBSE. Scoliosis 2008;3:8

31. Geissele AE, Kransdorf MJ, Geyer CA, et al. Magnetic resonance imaging of the brain stem in adolescent idiopathic scoliosis. Spine (Phila Pa 1976) 1991;16:761-63

32. Dohn $\mathrm{P}$, Vialle R, Thevenin-Lemoine $\mathrm{C}$, et al. Assessing the rotation of the spinal cord in idiopathic scoliosis: a preliminary report of MRI feasibility. Childs Nerv System 2009;25:479-83

33. Porter RW. Can a short spinal cord produce scoliosis? Eur Spine J 2001;10:2-9

34. Mohammadi S, Freund P, Feiweier T, et al. The impact of post-processing on spinal cord diffusion tensor imaging. Neuroimage 2013;70:377-85

35. Petersen JA, Wilm BJ, von Meyenburg J, et al. Chronic cervical spinal cord injury: DTI correlates with clinical and electrophysiological measures. J Neurotrauma 2012;29:1556-66

36. Ellingson BM, Kurpad SN, Schmit BD. Functional correlates of diffusion tensor imaging in spinal cord injury. Biomed Sci Instrum 2008;44:28-33

37. Ellingson BM, Kurpad SN, Schmit BD. Characteristics of mid- to long-latency spinal somatosensory evoked potentials following spinal trauma in the rat. J Neurotrauma 2008;25:1323-34 